University of Nebraska - Lincoln

DigitalCommons@University of Nebraska - Lincoln

April 2000

\title{
Manganese surface segregation in NiMnSb
}

\author{
Delia Ristoiu \\ CNRS Laboratoire Louis Nee'l
}

J. P. Nozie`res

CNRS Laboratoire Louis Nee'l, 25 Avenue des Martyrs BP 166, 38042 Grenoble CEDEX 09, France

C.N. Borca

University of Nebraska-Lincoln

B. Borca

University of Nebraska-Lincoln

Peter A. Dowben

University of Nebraska-Lincoln, pdowben@unl.edu

Follow this and additional works at: https://digitalcommons.unl.edu/physicsdowben

Part of the Physics Commons

Ristoiu, Delia; Nozie`res, J. P. ; Borca, C.N.; Borca, B.; and Dowben, Peter A., "Manganese surface segregation in NiMnSb" (2000). Peter Dowben Publications. 30.

https://digitalcommons.unl.edu/physicsdowben/30

This Article is brought to you for free and open access by the Research Papers in Physics and Astronomy at DigitalCommons@University of Nebraska - Lincoln. It has been accepted for inclusion in Peter Dowben Publications by an authorized administrator of DigitalCommons@University of Nebraska - Lincoln. 


\title{
Manganese surface segregation in NiMnSb
}

\author{
Delia Ristoiu and J. P. Nozières \\ CNRS Laboratoire Louis Neél, 25 Avenue des Martyrs BP 166, 38042 Grenoble CEDEX 09, France
}

C. N. Borca, B. Borca, and P. A. Dowben ${ }^{\text {a) }}$

Department of Physics and Astronomy and the Center for Materials Research and Analysis, Behlen Laboratory of Physics, University of Nebraska-Lincoln, Lincoln, Nebraska 68588-0111

(Received 21 December 1999; accepted for publication 25 February 2000)

\begin{abstract}
A quantitative analysis of the surface composition of the Heusler alloy NiMnSb has been undertaken using angle-resolved x-ray photoemission spectroscopy and the surface composition characterized. With sufficient annealing cycles, the stoichiometric surface evolves to a surface that is manganese rich. This indicates that the surface enthalpy is different from the bulk for the Heusler alloy NiMnSb. () 2000 American Institute of Physics. [S0003-6951(00)00617-3]
\end{abstract}

The presence of surface segregation is an indicator of a difference in free energy (chemical potential) between the surface and the bulk in the absence of segregation. ${ }^{1,2}$ This difference in energy can be related to the creation of a surface electronic structure very different from the bulk, as has been noted for the complex oxides, ${ }^{2-4}$ as well as result in a surface lattice structure different from the bulk and, of course, become the driving force for segregation. In addition, defects and variations in the local lattice order are believed to strongly influence the net electron polarization in $\mathrm{NiMnSb}$ near the Fermi level. ${ }^{5}$

We have been able to establish that surface preparation has a profound effect upon the surface electronic structure and polarization in spin-polarized inverse photoemission for the Heusler alloy NiMnSb. ${ }^{6}$ The possibility of surface segregation has been suggested for NiMnSb. ${ }^{7,8}$ Bona et al. proposed that $\mathrm{Sb}$ segregation dominates the surface region. ${ }^{7} \mathrm{In}$ fact, as we show in this letter, once the excess Sb protective capping layer is removed, Mn segregation dominates the surface and near surface region of NiMnSb.

The single crystal epitaxial thin film $\mathrm{NiMnSb}(100)$ samples were grown on a $\mathrm{Mo}(100)$ seed layer grown on chemically cleaned $\mathrm{MgO}(100)$ substrate. A $1000 \AA$ Sb capping layer was deposited to prevent oxidation of the NiMnSb crystalline films prior to the surface sensitive measurements. Crystallinity and orientation of the NiMnSb was established by $x$-ray diffraction and, once the capping layers were removed, again by low-energy electron diffraction (LEED).

Angle-resolved x-ray photoemission spectroscopy (ARXPS) of the $\mathrm{Sb}, \mathrm{Mn}$, and Ni core levels was undertaken on a number of different samples. The elemental $\mathrm{Ni}\left(2 p_{3 / 2}\right)$, $\operatorname{Mn}\left(2 p_{3 / 2}\right.$ and $\left.2 p_{1 / 2}\right)$, and $\mathrm{Sb}\left(3 d_{5 / 2}\right.$ and $\left.3 d_{3 / 2}\right)$ core levels and the intensities were measured, as were the binding energies, as a function of emission angle, with respect to the surface normal, using a (PHI Model 10-360 hemispherical energy analyzer).

Core level photoemission data are shown for $\mathrm{NiMnSb}$ in Fig. 1. The Mn $2 p_{3 / 2}$ spectra from the NiMnSb samples and the LEED (insert to Fig. 1) indicate that the disorder is limited in the surface region. The full width at half maximum of the $\mathrm{Mn} 2 p_{3 / 2}$ core level spectra is 5.3 to $5.8 \mathrm{eV}$ as compared

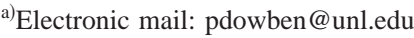

to $4-4.5 \mathrm{eV}$ for $\mathrm{La}_{0.65} \mathrm{Ca}_{0.35} \mathrm{MnO}_{3}$ and $\mathrm{La}_{0.65} \mathrm{Sr}_{0.35} \mathrm{MnO}_{3}{ }^{9}$ The increase in the $\mathrm{Mn} 2 p_{3 / 2}$ half width is consistent with the placement of $\mathrm{Mn}$ in a more metallic environment in half metallic NiMnSb than is the case in the half metallic manganese perovskites. (The core level line shape does not provide a good indicator as to the defect density in the surface region.)

Following different surface preparation procedures, we have plotted the emission angle dependent XPS core level intensity ratios in Fig. 2. The measured XPS intensity $I(\theta)$,

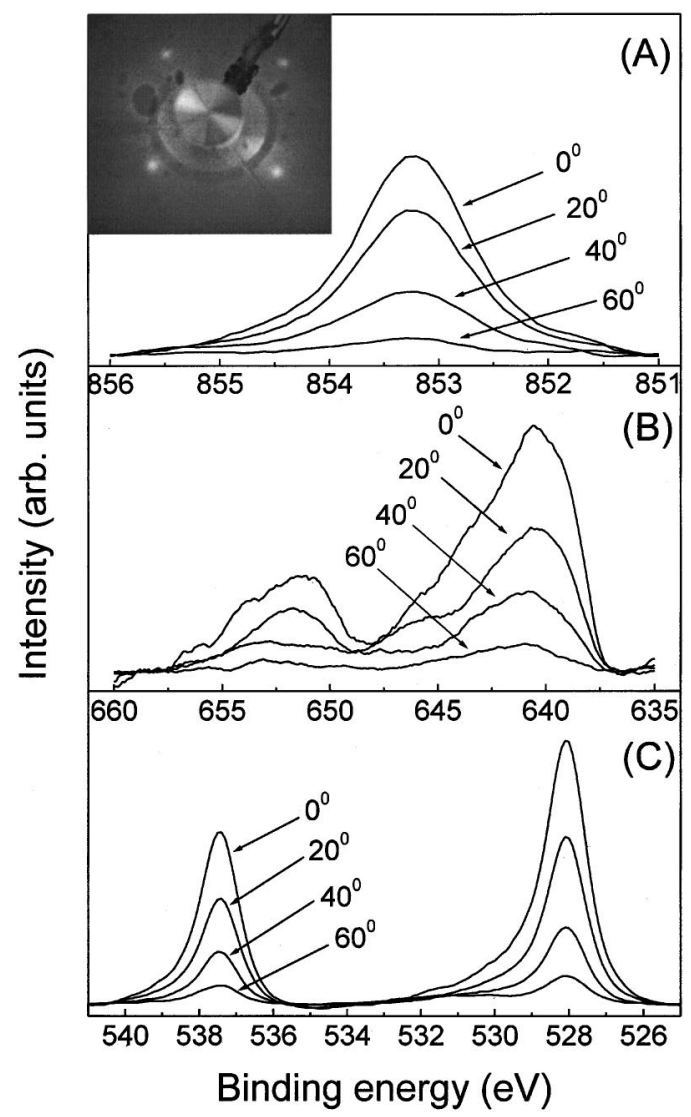

FIG. 1. The emission angle dependence of the $\mathrm{x}$-ray photoemission data. The Ni $2 p_{3 / 2}(\mathrm{~A}), \mathrm{Mn} 2 p_{3 / 2}+2 p_{1 / 2}(\mathrm{~B})$, and $\mathrm{Sb} 3 d_{5 / 2}+3 d_{3 / 2}(\mathrm{C})$ core levels of NiMnSb are shown as a function of emission angle, as indicated. The LEED pattern of the stoichiometric surface for $\mathrm{NiMnSb}$, obtained at an electron energy of $34 \mathrm{eV}$ is shown in the inset. 


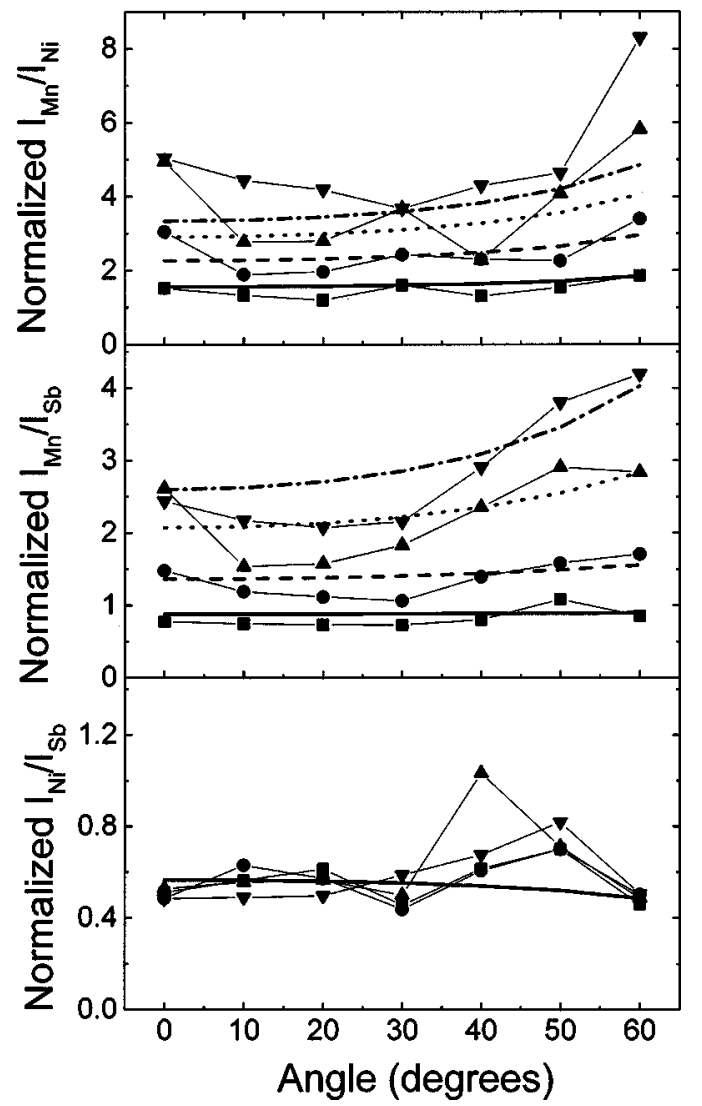

FIG. 2. The angle resolved $x$-ray photoemission intensity ratios of the $\mathrm{Ni} 2 p_{3 / 2}, \mathrm{Mn} 2 p_{3 / 2}$, and $\mathrm{Sb} 3 d_{5 / 2}$ core levels of NiMnSb. The data are compiled from spectra were taken as a function of emission angle following removal of the excess $\mathrm{Sb}$ with a $700 \mathrm{~K}$ anneal (ם) and following continued sputtering and annealing cycles of $10 \mathrm{~min}(\boldsymbol{\cup})$ followed by $1 / 2 \mathrm{~h}(\boldsymbol{\Delta})$ and finally by $1 \mathrm{~h}(\boldsymbol{\nabla})$ total annealing times. The data points are fitted with a segregation model in which $\mathrm{Mn}$ atoms migrate to both vacancy sites as well as replace $\mathrm{Sb}$ and $\mathrm{Ni}$ atoms from their lattice sites in the surface region except for data for the surface stoichiometric alloy termination MnSb (surface): $\mathrm{Ni}$ : $(\mathrm{MnSb}: \mathrm{Ni})_{n}$. The details of the $\mathrm{Mn}$ concentration profiles used to model the data are shown in Fig. 3(a).

for the different elemental core levels ( $2 p_{3 / 2}$ for $\mathrm{Ni}$ and $\mathrm{Mn}$, $3 d_{5 / 2}$ for $\mathrm{Sb}$ ) is normalized by the cross section for the different elemental core levels and the transmission factor. The normalized intensity ratio is thus given as

$$
R(\theta)=\left[\frac{I_{A}(\theta) / \sigma_{A}}{I_{B}(\theta) / \sigma_{B}}\right]\left[\frac{E_{\mathrm{kin}}^{p}(A)-C}{E_{\mathrm{kin}}^{p}(B)-C}\right],
$$

where $\theta$ is the emission angle with respect to the surface normal, $\sigma_{A}, \sigma_{B}$ are the cross sections [using the calculations by Scofield for an excitation energy of $\left.1253.6 \mathrm{eV}\left(\mathrm{Mg} K_{\alpha}\right)\right]^{10}$ and the term $E_{\mathrm{kin}}^{p}(A)-C$ corrects for the transmission of the electron energy analyzer at the kinetic energy of core level $A$. Based on the measured transmission functions for this type of analyzer, ${ }^{11}$ we have set $p=0.5, C=0$.

A procedure that results in the preparation of the stoichiometric or near stoichiometric ordered alloy surface, terminating in $\mathrm{MnSb}$, was outlined previously. ${ }^{6} \mathrm{Ar}^{+}$sputtering (to remove surface contamination) followed by a flash anneal to $700 \mathrm{~K}$ (to remove the excess $\mathrm{Sb}$ ) results in one of the normalized XPS intensity ratios sets shown in Fig. 2. This surface exhibits a sharp LEED pattern (as seen in the inset to Fig. 1), with a $6.0 \pm 0.1 \AA$ surface lattice constant consistent with the $5.9 \AA$ unit cell of NiMnSb.

Downloaded 06 Sep 2006 to 129.93.16.206. Redistribution subject to AlP license or copyright, see http://apl.aip. $\lambda_{\mathrm{Ni}}$ (

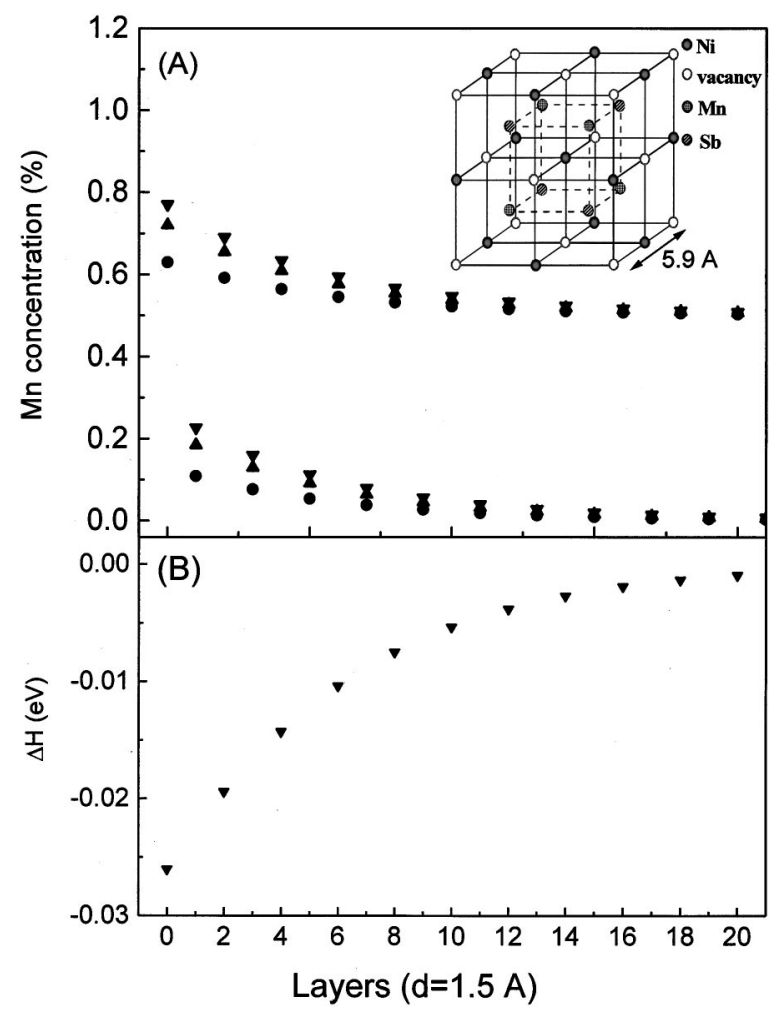

FIG. 3. The concentration of $\mathrm{Mn}$ in the near surface region obtained from fits from the angle resolved x-ray photoemission data are shown in panel (a). The odd layers are $\mathrm{Mn} / \mathrm{Ni}$. The $\mathrm{Mn}$ concentration profiles denoted by the different symbols $(\boldsymbol{\Lambda} \boldsymbol{\nabla})$ are fit to the data shown by similar symbols in Fig. 2. The energy difference between the surface and the bulk based on the most extensive Mn segregation is shown in panel (b). The inset shows the crystal structure of NiMnSb.

As previously noted, ${ }^{6}$ the surface of $\mathrm{NiMnSb}$ is fragile and $\mathrm{Mn}$ segregation readily occurs, as indicated by the angle resolved x-ray photoemission shown in Fig. 2. With continued annealing (and sputtering to remove surface contamination), there is a clear sequence of increasing $\mathrm{Mn} / \mathrm{Sb}$ and $\mathrm{Mn} / \mathrm{Ni}$ ratios, though the $\mathrm{Ni} / \mathrm{Sb}$ core level intensity ratio is largely preserved.

The different sets of data can be analyzed using a previously applied semi-empirical method ${ }^{1,2}$ to give a more quantitative picture of the extent of surface segregation. Quantitative analysis of the data makes use of the decrease in the effective mean free path of the escaping electrons with increasing emission angle. ${ }^{1,2}$ A summation is undertaken to account for each layer contributing to the photoemission signal. NiMnSb has, effectively, a layered crystal structure (inset to Fig. 3) with alternate $\mathrm{Mn}-\mathrm{Sb}$ and Ni-vacancy layers, with the distance between layers approximately $d$ $=a_{\mathrm{NiMnSb}} / 4=1.5 \AA$. The various models for surface termination with no segregation would have $f_{j}(A), f_{j}(B)=1 / 2$ or zero depending upon the layer considered, for components $A$ and $B$ (since we have two elements per layer). Thus, for the constituent component $A$, with concentration $f_{j}(A)$, the normalized core level intensities can be written with respect to a reference constituent component $B$, as

$$
R(\theta)=\frac{\sum_{j=0}^{\infty} f_{j}(A) e^{-j d /\left[\lambda_{A} \cos (\theta)\right]}}{\sum_{j=0}^{\infty} f_{j}(B) e^{-j d /\left[\lambda_{B} \cos (\theta)\right]}},
$$

where $\lambda_{\mathrm{Ni}}\left(7 \AA\right.$ for the Ni $2 p_{3 / 2}$ core), $\lambda_{\mathrm{Mn}}(9.35 \AA$ for the 
Mn $2 p$ cores), and $\lambda_{\mathrm{Sb}}(10.8 \AA$ for the $\mathrm{Sb} 3 d$ cores $)$ are the effective mean free paths, adapted from the calculated mean free paths of Penn. ${ }^{12}$ The surface, with the Sb cap removed and little additional annealing, can be modeled using Eq. (2). We find that models that have the surface terminate in $\mathrm{NiSb}$ or NiMn do not fit the data nearly as well as the model based upon the stoichiometric alloy terminating in $\mathrm{MnSb}$ at the surface. $^{6}$
Because the core level photoemission intensity ratios change with increased annealing, as seen in Fig. 2, the models for the core level intensity must include the possibility of segregation. For Mn segregation, we postulate that Mn segregation to the surface and to the vacancy sites within the $\mathrm{Ni}$ layers are both possible. To account for this type of segregation we have fit the data in Fig. 2 with the normalized intensity ratio of Mn to a reference constituent $B$ rewritten as

$$
R(\theta)=\frac{\sum_{j=0}^{\infty}\left\{\frac{1}{2} e^{-[(2 j+1) d] /\left[\lambda_{A} \cos (\theta)\right]}+\delta e^{-(2 j / G)} e^{-(2 j d) /\left[\lambda_{A} \cos (\theta)\right]}\right\}}{\sum_{j=0}^{\infty} \frac{1}{2} e^{-(2 j+\xi) d /\left[\lambda_{B} \cos (\theta)\right]}},
$$

where the parameters $\delta$ and $G$ are the segregation to the topmost $(j=0)$ layer (above the terminal MnSb layer) and the segregation depth in units of the distance $d$ between layers, respectively. Thus MnSb layers are the odd numbered layers and $\mathrm{Ni}$ plus segregated Mn occupy even layers except for the zeroth layer which contains only Mn segregation. Alternatively Mn can segregate to both vacancy sites as well as replace both $\mathrm{Sb}$ and $\mathrm{Ni}$, in this scheme

$$
R(\theta)=\frac{\sum_{j=0}^{\infty}\left(\exp \left[\frac{-2 j d}{\lambda_{A} \cos (\theta)}\right]+\delta\left\{\exp \left[\frac{-2 j}{G}+\frac{-2 j d}{\lambda_{A} \cos (\theta)}\right]+\exp \left[\frac{-(2 j+1)}{G}+\frac{-(2 j+1) d}{\lambda_{A} \cos (\theta)}\right]\right\}\right)}{\sum_{j=0}^{\infty}\left\{\exp \left[\frac{-(2 j+\xi) d}{\lambda_{B} \cos (\theta)}\right]-\delta \exp \left[\frac{-(2 j+\xi)}{G}+\frac{-(2 j+\xi) d}{\lambda_{B} \cos (\theta)}\right]\right\}} .
$$

So $\xi=1$ for Ni the reference and $\xi=0$ for $\mathrm{Sb}$ the reference. The surfaces exhibiting the larger $\mathrm{Mn} / \mathrm{Sb}$ and $\mathrm{Mn} / \mathrm{Ni}$ ratios can be modeled if Mn segregation is considered, using (4), as seen in Fig. 2 by the solid line indicating the model fits. The model fits to the data are shown in Fig. 3(a) and compared to the experimental data (following increasingly extensive segregation) in Fig. 2.

From the fits to the data, we have constructed Mn concentration profiles [see Fig. 3(a)] from the surface to the bulk using the formulas $f_{i}(\mathrm{Mn})=b+\delta(\exp -j / G)$, where $b=1 / 2$ for the MnSb layers and $b=0$ for the Ni layers. With increased annealing (and sputtering) at $700 \mathrm{~K}$, the changing core level intensity ratios can be roughly modeled by $\mathrm{Mn}$ segregation to the surface, Mn segregation to the vacancy sites, and Mn segregation possibly leading to the displacement of atoms for the extensively annealed sample, as shown in Fig. 3(a) and indicated by the symbol $(\boldsymbol{\nabla})$. The extensive annealing leads to formation of a different material from $\mathrm{NiMnSb}$ in the surface region. Because our model fits are not unique solutions to the data, the exact details of the surface structure with extensive annealing of $\mathrm{NiMnSb}$ will require other, surface sensitive, structural studies.

The substantial surface segregation implies an energy difference (in the absence of segregation). ${ }^{13}$ This has been calculated from the segregation profiles, as in Fig. 3(b) using the approximation that $f_{j}(A) / f_{j}(B)=\left[f_{b}(A) / f_{b}(B)\right] \exp$ $\left(-\Delta H / k_{B} T\right)$, where $f_{b}(A)$ and $f_{b}(B)$ are the bulk layer concentrations of constituents $A$ and $B$, and $T$ is the annealing temperature.

The extensive segregation of manganese would reduce the measured polarization of the surface given the influence of atomic disorder on the electronic structure. ${ }^{5}$ The observed segregation, noted here, is very consistent with marked decline in polarization measured in spin-polarized inverse photoemission following extensive segregation ${ }^{6}$ as well as in spin-polarized tunneling measurements. ${ }^{14}$

This work was supported by NSF through Grant No. DMR-98-02126, the Center for Materials Research and Analysis (CMRA), the Nebraska Research Initiative at the University of Nebraska, and the Region Rhone-Alpes through the "Nanotechnologie" program under Contract No. PR97024.

${ }^{1}$ Surface Segregation Phenomena, edited by P. A. Dowben and A. Miller (CRC Press, Boston, 1990), p. 145.

${ }^{2}$ J. Choi, C. Waldfried, S.-H. Liou, and P. A. Dowben, J. Vac. Sci. Technol. A 16, 2950 (1998); J. Choi, J. Zhang, S.-H. Liou, P. A. Dowben, and E. W. Plummer, Phys. Rev. B 59, 13453 (1999).

${ }^{3}$ S. H. Liu and R. A. Klemm, Phys. Rev. Lett. 73, 1019 (1994).

${ }^{4}$ M. Lindroos and A. Bansil, Phys. Rev. Lett. 75, 1182 (1995).

${ }^{5}$ D. Orgassa, H. Fujiwara, T. C. Schulthess, and W. H. Butler, Phys. Rev. B 60, 13237 (1999).

${ }^{6}$ D. Ristoiu, J. P. Nozières, C. N. Borca, T. Komesu, H. K. Jeong, and P. A. Dowben, Europhys. Lett. (in press).

${ }^{7}$ G. L. Bona, F. Meier, M. Taborelli, E. Bucher, and P. H. Schmidt, Solid State Commun. 56, 391 (1985).

${ }^{8}$ J.-H. Park, E. Vescovo, H.-J. Kim, C. Kwon, R. Ramesh, and T. Venkatesan, Nature (London) 392, 794 (1998).

${ }^{9}$ J. Choi, H. Dulli, S.-H. Liou, P. A. Dowben, and M. A. Langell, Phys. Status Solidi B 214, 45 (1999).

${ }^{10}$ J. H. Scofield, J. Electron Spectrosc. Relat. Phenom. 8, 129 (1976).

${ }^{11}$ M. P. Seah, M. E. Jones, and M. T. Anthony, Surf. Interface Anal. 6, 242 (1984); M. P. Seah, ibid. 20, 243 (1993).

${ }^{12}$ D. R. Penn, J. Electron Spectrosc. Relat. Phenom. 9, 29 (1976).

${ }^{13}$ S. Y. Liu and H. H. Kung, Surf. Sci. 110, 504 (1981).

${ }^{14}$ C. T. Tanaka, J. Nowak, and J. S. Moodera, J. Appl. Phys. 86, 6239 (1999). 